\title{
Millimeter-wave antenna measurement
}

\author{
J.A.G. Akkermans ${ }^{\# 1}$, R. van Dijk ${ }^{* 2}$, M.H.A.J. Herben ${ }^{\# 3}$ \\ \# Radiocommunications group, Eindhoven, University of Technology \\ Eindhoven, The Netherlands \\ ${ }^{1} \mathrm{j} \cdot \mathrm{a} \cdot \mathrm{g} \cdot \mathrm{akkermans}$ atue.nl \\ ${ }^{3} \mathrm{~m} \cdot \mathrm{h} \cdot \mathrm{a} \cdot \mathrm{j} \cdot \mathrm{herben}$ tue.nl \\ ${ }^{*} T N O$ Defence, Security and Safety \\ The Hague, The Netherlands \\ ${ }^{2}$ raymond. vandijketno.nl
}

\begin{abstract}
A novel approach is presented to accurately measure the scattering parameters as well as the radiation pattern of planar antennas that operate in the millimeter-wave frequency band. To avoid interconnection problems, RF probes have been used to connect to the antenna. These RF probes are normally used for the characterisation of devices on a semiconductor wafer. Here, they are used to characterise antennas that can be realised in any planar manufacturing technology. A completely planar transition is designed to transform the coplanar waveguide transmission line that is required by the $\mathrm{RF}$ probe to a microstrip line. It is shown that this transition can be de-embedded from the measurements. Moreover, a measurement setup has been built for the measurement of the far-field radiation pattern of millimeterwave antennas and antenna arrays. This setup allows to connect the antenna under test with an RF probe and can measure the radiation pattern in the whole upper hemisphere.
\end{abstract}

\section{INTRODUCTION}

An approach is presented that allows for the accurate characterisation of the performance of planar antennas that operate in the millimeter-wave frequency band. In order to avoid interconnection problems, RF probes have been used such that a well-defined interconnection between the antenna under test (AUT) and the measurement setup can be realised. RF probes have the advantage that they are reusable and that they can be calibrated very accurately.

A completely planar transition is designed to transform the coplanar waveguide transmission line that is required by the $\mathrm{RF}$ probe to a microstrip transmission line that is used by the antenna structure. It is shown that this transition can be de-embedded from the measurements. A measurement setup has been realised to measure the far-field radiation pattern as well. It is based on a measurement setup that was proposed by [1] and is optimised such that the setup does not affect the measured radiation pattern. It allows the measurement of the far-field radiation pattern in the whole upper hemisphere.

The AUT that has been measured is a balanced-fed aperturecoupled patch antenna [2]. This antenna operates in the band from 54 to $60 \mathrm{GHz}$ and has been realised in printed circuitboard technology. In [2] the focus was on the design of the antenna. Here, the focus lies on the measurement of the scattering parameters and the radiation pattern of this antenna.

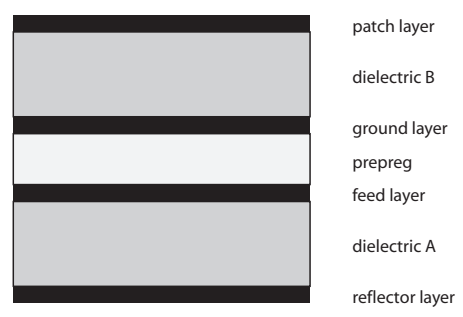

Fig. 1. PCB stack.

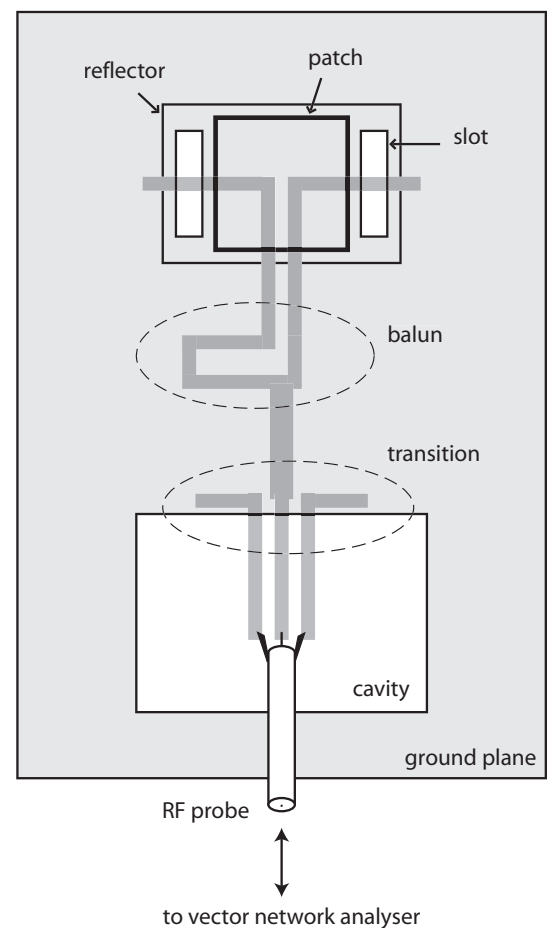

Fig. 2. Schematic layout of AUT and measurement setup.

\section{AUT AND MEASUREMENT SETUP}

The antenna is realised in a standard printed circuit-board (PCB) manufacturing technology from two dielectric boards that are stacked with a prepreg layer in between (Fig. 1). The feed lines are embedded within the stack. Therefore a cavity has been made such that the RF probe can be connected to 


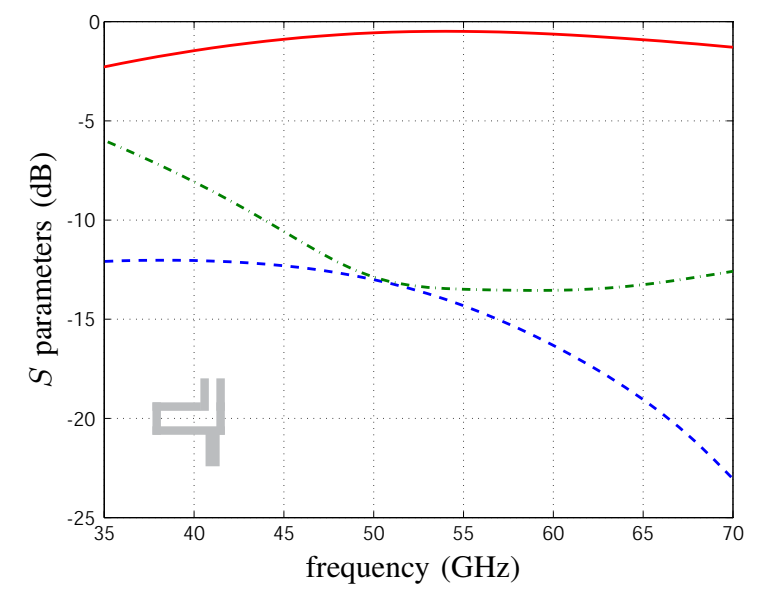

Fig. 3. Simulated scattering parameters of the balun. $S_{11}$ MS (dashed), $S_{12}$ MS - CPS [odd mode] (solid), $S_{22}$ CPS [odd mode] (dash-dot).

the feed lines. The cavity has been realised by creating a hole in the upper dielectric board and in the prepreg board before the boards are stacked together.

A schematic layout of the AUT and the measurement setup is shown in Figure 2. Since the antenna has a balanced feed, a balun is required which transforms the balanced coupled microstrip line to an unbalanced microstrip line. The RF probe has to land on a coplanar waveguide (CPW) transmission line. In order to connect to the balun, a transition from $\mathrm{CPW}$ to microstrip (MS) is needed. This transition is realised without the use of vias to avoid manufacturing uncertainties that are involved with the realisation of vias. Two quarter-wave stubs are attached to both ground lines of the CPW to provide a virtual ground at the end of the CPW line, following an idea by Raskin et al. [3].

The balun design is quite straightforward. The microstrip line connects to two lines that have a length difference which corresponds to 180 degrees of phase difference at $60 \mathrm{GHz}$. These two lines are immediately connected to the coplanar microstrip (CPS) transmission line that is used to feed the antenna. The CPS transmission line can support two modes, the odd mode and the even mode. The balun should excite the odd mode for the antenna to work properly. Simulation results shows that this is the case indeed and that the balun has a good performance over a wide frequency band (Fig. 3). These simulation results have been obtained with Ansoft Designer, which is a planar electromagnetic field simulator.

\section{DE-EMBEDDING}

A challenge in the measurement of the reflection coefficient of the antenna is to de-embed the CPW to MS transition from the measurements. For this purpose, additional structures have been realised on the antenna board. Three different types of transitions in a back-to-back configuration have been realised, i.e., an open, thru and line configuration (Fig. 4). In this way, all the S-parameters of the transition can be characterised following a TRL calibration procedure [4].

The performance of the transition is shown in Fig 5. It

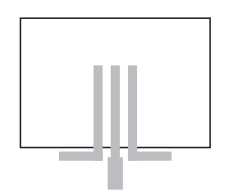

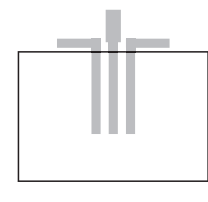

open
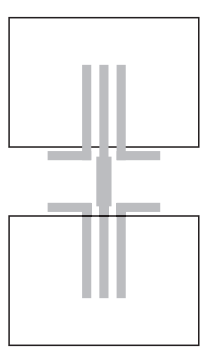

thru

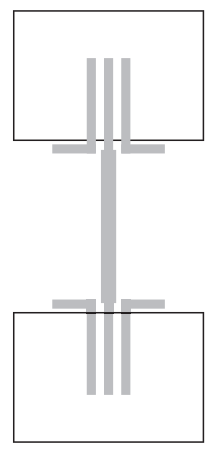

line
Fig. 4. Open, thru and line configurations for characterisation of the CPW to MS transition.

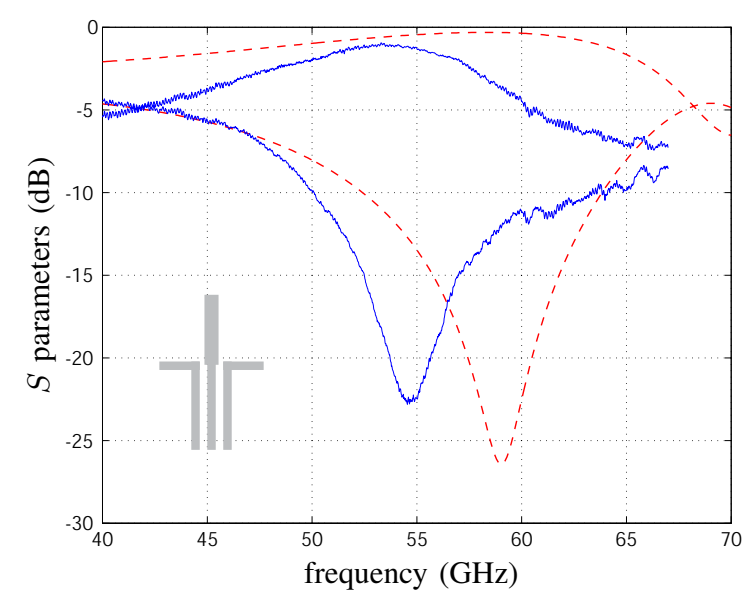

Fig. 5. Scattering parameters of the CPW-MS transition. measurement (solid), simulation (dashed).

is observed that the performance of the realised transition is somewhat different than the performance of the simulated transition. This discrepancy is mainly caused by two factors. The first factor is the uncertainty in the material properties. The material properties of the dielectric boards were specified up to $10 \mathrm{GHz}$ and the material properties of the prepreg layer were specified up to $40 \mathrm{GHz}$. In the simulations, these values have been used for the characterisation of the structure at $60 \mathrm{GHz}$. A negative frequency shift is observed in the measurements. Therefore it is concluded that the permittivity of the dielectric boards is somewhat higher than the permittivity that is specified at $10 \mathrm{GHz}$. The second uncertainty factor is the thickness of the prepreg layer. The boards are stacked under pressure, which introduces some tolerance on the thickness of the prepreg layer. The test structures and the antenna have been processed on the same board such that the layer thicknesses of all structures are similar. At this point, it is important to stress that although the structures have not exactly the expected performance, it is still possible to accurately de-embed the effect of the transition from the measurements, since the deembedding procedure relies on the measured transition only.

The measured and simulated reflection coefficients of the antenna and balun after de-embedding are shown in Figure 6. 


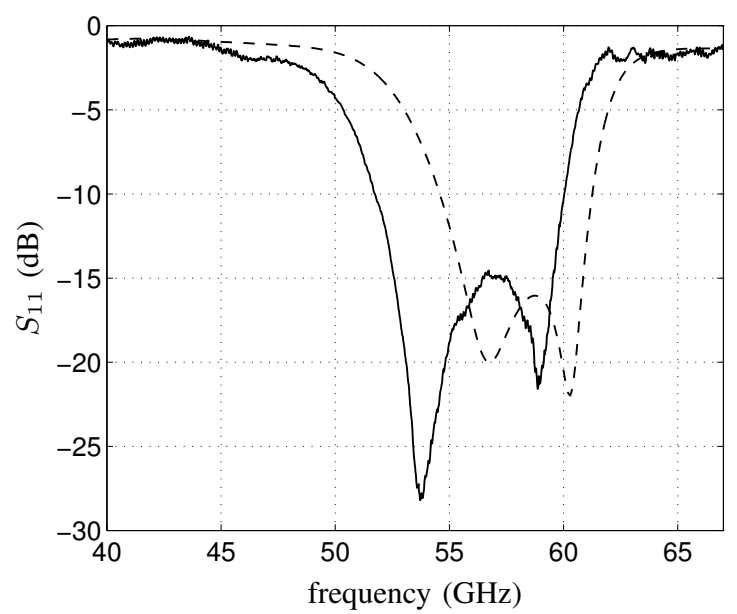

Fig. 6. Measured (solid) and simulated (dash) reflection coefficient of the antenna and balun.

A good agreement between measurement and simulation is observed although the operation frequency of the antenna is somewhat shifted. This is partly due to differences between the expected and the actual material properties of the dielectric boards. Simulations have shown that the tolerances on the thickness of the prepreg layer affects the input impedance of the antenna very little.

\section{RADIATION PATTERN}

\section{A. Measurement setup}

To determine the radiation pattern of the antenna, a far-field measurement setup has been built (Fig. 7). It is based on the setup that was proposed in [1], which measures the radiation pattern of patch antennas that operate in the frequency range around $24 \mathrm{GHz}$. In [1], the radiation pattern has been measured above a probe station, which can introduce a lot of unwanted scattering due to the metal structure of the probe station.

Our setup is tailored for the measurement of the radiation pattern of millimeter-wave antennas and antenna arrays. It is tried to minimise the scattering of the measurement setup itself. The measurement setup has a metal base which will be covered with absorber. The antenna is placed on a perspex table and a rigid perspex arm is used that supports the measurement probe (see Fig. 7). This light-weight arm can be rotated in elevation by a stepper motor and can be placed at different azimuth angles to be able to measure the full hemisphere. An open-ended waveguide is attached to the end of the perspex arm to measure the far-field radiation. The antenna is connected with an RF probe. In order to land this $\mathrm{RF}$ probe on the contact pads of the antenna, a micro positioner is needed. This positioner is placed at the back of the metal base to reduce the scattering and has a perspex bar that moves through a hole in the metal base.

In order to position the center of the antenna in the rotational center of the measurement arm, a digital video camera is used that can be placed at a fixed position above the AUT. Since the camera has a fixed position, the rotational center of the

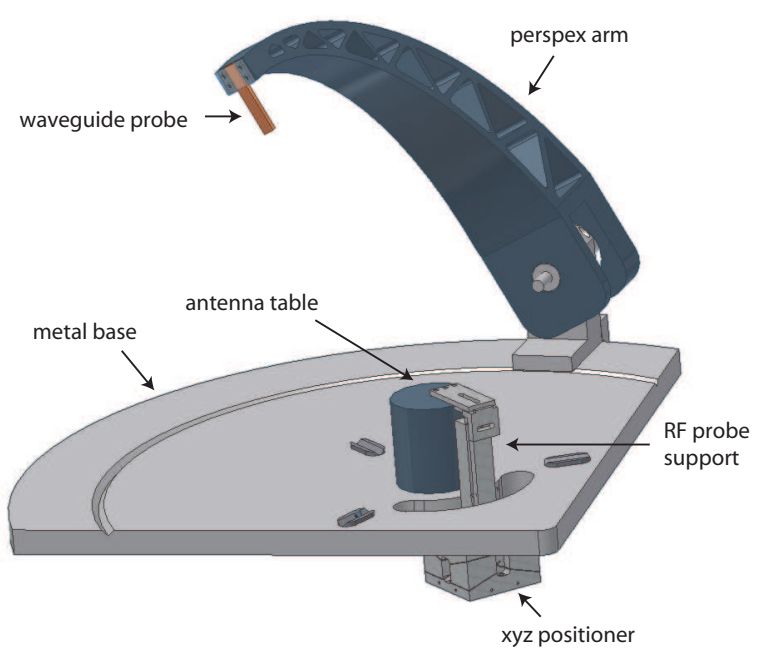

Fig. 7. Setup for radiation pattern measurement.

measurement setup can be displayed on the camera view and the antenna under test can be moved such that its center and the rotational center of the measurement setup coincide.

The open-ended waveguide can be positioned in three different rotations $(0,45,90$ degrees) with respect to the perspex arm. In this way, the co- and cross-polar radiation patterns can be determined in the principle $\mathrm{E}$ and $\mathrm{H}$ plane and in the diagonal plane with a measurement of the magnitude of the radiated field only. For these measurements, no phase information is needed. In order to determine the radiated field in the whole upper hemisphere, measurements of the magnitude and phase of the radiated field is required in two different polarisations.

The far-field region of regular antennas in the millimeterwave frequency bands starts at a distance in the order of tens of centimeters from the antenna. Therefore the setup that we propose is very much suited for such measurements, since it is possible to characterise the antenna with a setup which has a limited size and which allows for a simple interconnection with the antenna under test. The open-ended waveguide is placed at a distance of about 30 centimeters from the AUT. The far-field distance $r_{\text {ff }}$ can be estimated as (see e.g. [5])

$$
r_{\mathrm{ff}}=\frac{2 D^{2}}{\lambda}
$$

with $D$ the largest dimension of the antenna and $\lambda$ the freespace wavelength. Equation 1 implies a maximum dimension of the antenna of about $27 \mathrm{~mm}$ for an operation frequency of $60 \mathrm{GHz}$.

\section{B. Measurements}

The radiation pattern of the antenna has been measured in the frequency range from 50 to $60 \mathrm{GHz}$. The antenna table has been covered with absorbing material and a polystyreen layer has been placed underneath the antenna. Polystyreen, has 


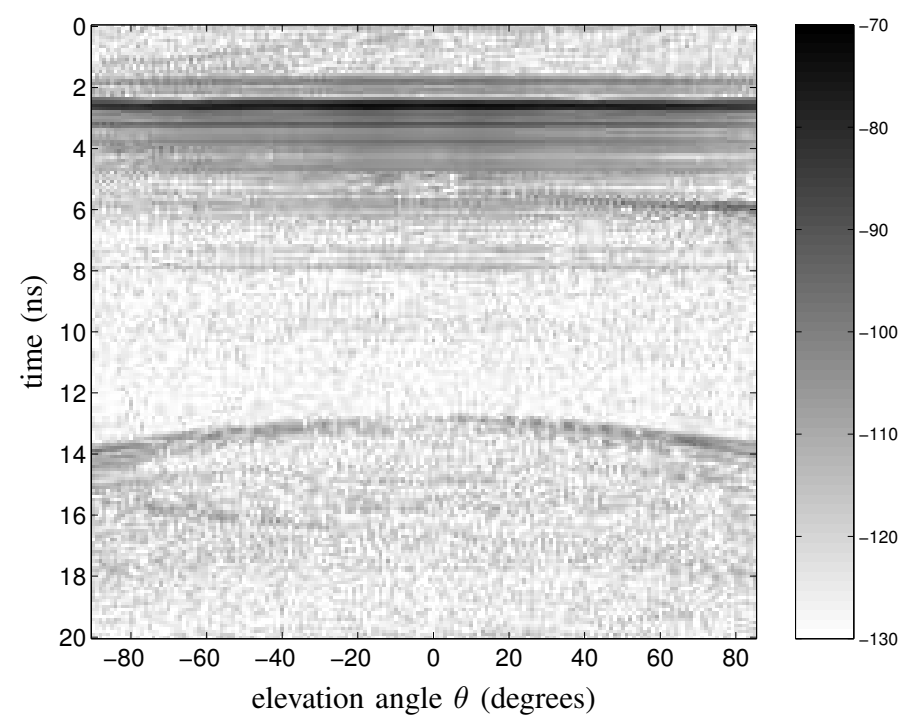

Fig. 8. Measured time-domain signals $(\mathrm{dB})$ as a function of elevation angle.

a relative permittivity close to one and therefore it does not affect the performance of the antenna. Since the metal body of the RF probe can distort the radiation pattern, it has been covered with absorbing material as well. The measurements have been conducted in a normal lab environment. To analyse the effect of the environment, the measured signals have been transformed to the time-domain. In Figure 8 the time domain signal is shown as a function of elevation angle $\theta$. It is seen that the maximum of the power is received around $t=2.5 \mathrm{~ns}$. Moreover, it is observed that there are some contributions which have a dependency on the elevation angle $\theta$. These contributions result from reflections of the environment. E.g., the measured signals around $t=14 \mathrm{~ns}$ are caused by reflections from the ceiling of the laboratory. To reduce the effect of the environment, time-gating has been applied. The time-domain signals are filtered from $t=1.8 \mathrm{~ns}$ to $t=4.9 \mathrm{~ns}$ and transformed to the frequency domain. The resulting radiation pattern is shown in Figure 9. It is observed that the agreement with the simulated radiation pattern is quite good. The simulations have been performed with CST Microwave Studio, which is a three dimensional finite-element electromagnetic simulator. In these simulations also the effect of the finiteness of the dielectric layers is included. The radiation from the edges of the dielectric causes dips in the radiation pattern around the elevation angles $\theta=-30,0$ and 30 degrees. It is interesting to note that this effect can be accurately measured as well.

\section{CONCLUSIONS}

A measurement setup has been proposed for the characterisation of planar antennas in the millimeter-wave frequency band. RF probes have been used such that a welldefined interconnection between the antenna under test and the measurement setup can be realised. A completely planar transition has been designed to transform the coplanar waveguide transmission line that is required by the RF probe to

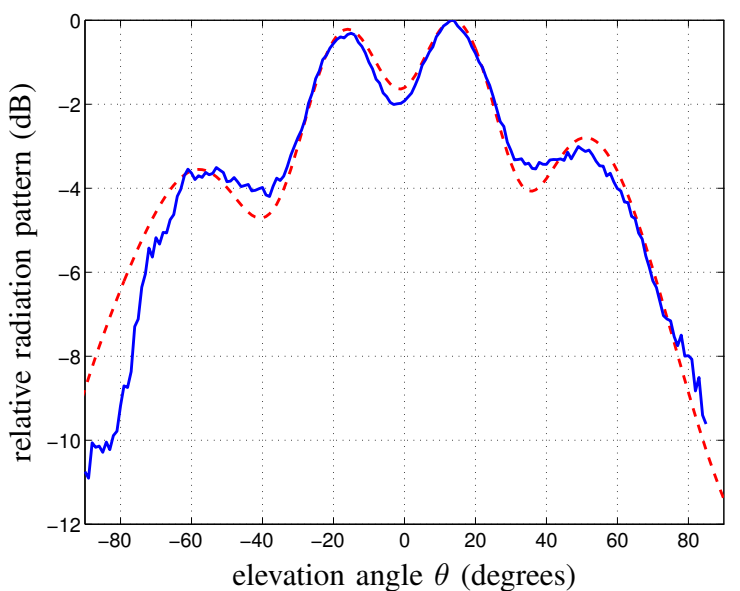

Fig. 9. Measured (solid) and simulated (dash) radiation pattern. E-plane, $f=54.2 \mathrm{GHz}$.

a microstrip line. This transition can be de-embedded from the measurements. Furthermore, a measurement setup has been realised to measure the far-field radiation pattern of the antenna under test in the whole upper hemisphere. The effect of the environment can be reduced by means of time gating. The measured radiation pattern is in good agreement with simulation, which confirms the validity of the proposed setup.

\section{REFERENCES}

[1] R. Simons, "Novel on-wafer radiation pattern measurement technique for mems actuator based reconfigurable patch antennas," Tech. Rep. NASA/TM-2002-211816, NASA, November 2002.

[2] J. Akkermans, M. van Beurden, and M. Herben, "Design of a millimeterwave balanced-fed aperture-coupled patch antenna," in proc. EuCAP 2006, ESA SP626, (Nice, France), pp. 1-6, November 2006.

[3] J.-P. Raskin, G. Gauthier, L. P. Katehi, and G. M. Rebeiz, "W-Band singlelayer vertical transitions," IEEE Transactions on Microwave Theory and Techniques, vol. 48, pp. 161-164, January 2000.

[4] D. Pozar, Microwave engineering. Wiley, 3rd ed., 2005

[5] W. Stutzman and G. Thiele, Antenna theory and design. John Wiley and Sons, Inc., 2nd ed., 1998. 